\title{
Electro-optic detection of subwavelength terahertz spot sizes in the near field of a metal tip
}

\author{
N. C. J. van der Valk ${ }^{\mathrm{a})}$ and P. C. M. Planken \\ Faculty of Applied Sciences, Department of Applied Physics, Delft University of Technology, Lorentzweg 1, \\ 2628 CJ, The Netherlands
}

(Received 6 May 2002; accepted for publication 9 July 2002)

\begin{abstract}
We report on a method to obtain a subwavelength resolution in terahertz time-domain imaging. In our method, a sharp copper tip is used to locally distort and concentrate the THz electric field. The distorted electric field, present mainly in the near field of the tip, is electro-optically measured in an (100) oriented GaP crystal. By raster scanning the tip along the surface of the crystal, we find the smallest $\mathrm{THz}$ spot size of $18 \mu \mathrm{m}$ for frequencies from 0.1 to $2.5 \mathrm{THz}$. For our peak frequency of $0.15 \mathrm{THz}$, this corresponds to a resolution of $\lambda / 110$. Our setup has the potential to reach a resolution down to a few $\mu \mathrm{m}$. (C) 2002 American Institute of Physics. [DOI: 10.1063/1.1503404]
\end{abstract}

In recent years, terahertz time-domain spectroscopy (TDS) has become a popular method to obtain information on the far-infrared properties of molecules, semiconductors, and other materials, ${ }^{1-4}$ and has shown to be a promising technique to image objects. ${ }^{5-7}$ Unfortunately, diffraction, which limits the resolution to about a wavelength, is an obstacle for a number of interesting new $\mathrm{THz}$ applications. Breaking the diffraction limit would, for instance, make it possible to perform $\mathrm{THz}$ spectroscopy on extremely small volumes, and, ultimately on living cells. Work on the label free probing of deoxyribonucleic acid (DNA), ${ }^{8}$ shows that $\mathrm{THz}$ radiation can be used to distinguish between different biomolecules.

One way to overcome the diffraction limit is to limit the detection area with an aperture. For example, Mitrofanov et $a l .{ }^{9}$ have shown that by using a detector with an integrated aperture, near field images can be made with a spatial resolution determined by the aperture size, and not by the $\mathrm{THz}$ wavelength. Unfortunately, this method relies on extensive micro-fabrication and requires two parallel plates, namely one carrying the object and the other the detector, to be scanned along each other at close distance, which is difficult to do in practice. Also, since the aperture has a finite thickness, waveguide effects strongly attenuate long-wavelength components, ${ }^{10}$ which makes their detection difficult. Another approach to obtain subwavelength resolution ${ }^{11}$ uses a laser to modulate the $\mathrm{THz}$ transmission of a small area on a semiconductor surface near the object. The modulation on the $\mathrm{THz}$ beam is measured in the far field, and an improved spatial resolution, down to $50 \mu \mathrm{m}$ is observed. In this method, however, signals are small and superimposed on a large background, complicating the detection.

Here, we present a method to create and detect a local $\mathrm{THz}$ source with a subwavelength resolution, which has the potential to image a microscopic object by raster scanning it between the source and the detector. In our method, $\mathrm{THz}$ pulses are incident on a sharp metal tip, having subwavelength dimensions, which is held in close proximity to an (100) oriented GaP crystal. A synchronized probe pulse is

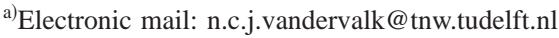

tightly focused into the crystal close to the tip and, using the electro-optic effect in the same crystal, only detects the $\mathrm{THz}$ polarization component that is perpendicular to the (100) surface. As the incident $\mathrm{THz}$ polarization is parallel to the crystal surface, the perpendicular component is present only near the tip, which functions as a lightning rod where field lines are bent and concentrated. We find that, directly under the tip, the measured THz spot size has a diameter of only 18 $\mu \mathrm{m}$, corresponding to $\lambda / 110$ for a wavelength of $2 \mathrm{~mm}$. Our method to obtain a subwavelength resolution has four advantages: (i) the resolution is determined by the spot diameter of our probe laser, not by the incident $\mathrm{THz}$ beam size, (ii) our measurements are background free, as we only detect the component near the tip that is perpendicularly polarized to the crystal surface, (iii) our detection setup measures in the near field, where the electric field can be strong. This provides sufficiently large signals and thus reduces the measurement time, and (iv) our method has the more practical requirement of scanning a tip along a surface, instead of two surfaces parallel to each other.

Our setup to generate $\mathrm{THz}$ pulses, is described elsewhere. ${ }^{12}$ As we show in Fig. 1, the THz pulses, which have an average power of $40 \mu \mathrm{W}$, are focused onto a copper tip, which has been sharpened in a $\mathrm{FeCl}$ etch bath. A $300 \mu \mathrm{m}$ thick, (100) oriented GaP detection crystal is placed within a distance of several microns of the tip. The crystal surface closest to the tip is coated with a $300 \mathrm{~nm}$ thick Ge layer on top off a $133 \mathrm{~nm}$ thick $\mathrm{SiO}_{2}$ layer. A synchonized, $800 \mathrm{~nm}$ probe pulse, derived from the same laser that generates the $\mathrm{THz}$ pulse, is focused to about a $20 \mu \mathrm{m}$ spot size on the coating, initially counter propagating with respect to the $\mathrm{THz}$ pulse. The coating, which has a negligible effect on the $\mathrm{THz}$ beam, absorbs any probe light before it reaches the tip. This eliminates any measurement errors that would have been caused by probe light scattered off the tip. After the reflection at the coating, the probe pulse is sent through a $\lambda / 4$ plate, and a Wollaston prism to a differential detector. When a $\mathrm{THz}$ electric field is present in the crystal, the electro-optic effect changes the probe polarization from linear to elliptical. The amount of ellipticity is proportional to the instantaneous $\mathrm{THz}$ electric field, and is measured by the differential detec- 


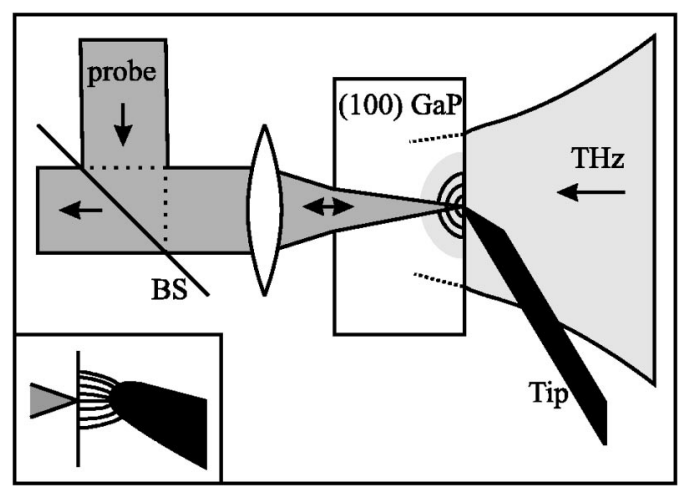

FIG. 1. Schematic picture of the setup used. The THz radiation comes in from the right-hand side, and scatters at the tip. The probe beam comes in from the left-hand side, and is focused on the crystal-air interface of an (100) $\mathrm{GaP}$ crystal. At this interface, the probe beam polarization is changed by the THz electric field near the tip. The probe beam reflects back at the crystal interface, and is sent to the differential detection setup (not shown). The inset on the bottom left-hand side zooms in on the tip, and schematically shows the near-field electric-field lines near the tip, which have a component perpendicular to the crystal surface.

tor setup. We emphasize that in an (110) oriented crystal, which is the conventionally used orientation in electro-optic detection, the probe polarization is only affected by electric fields parallel to the crystal surface. ${ }^{13}$ Here, however, using an (100) oriented crystal, the probe polarization is only changed by electric fields perpendicular to the crystal surface ${ }^{14}$ making the probe "blind" to the parallel polarized electric field of the incident $\mathrm{THz}$ pulse. Near the tip, however, as is illustrated in the inset of Fig. 1, the THz near field must have a component perpendicular to the crystal surface, since the electric field near a metal, in this case the tip, has to be perpendicular to the metal surface.

The inset of Fig. 2 shows the THz electric field as a function of the delay between the probe and $\mathrm{THz}$ pulse, measured directly under the copper tip, which is pressed against the crystal. The power spectrum of this pulse is plotted in Fig. 2. It contains frequencies from 0.1 to $2.5 \mathrm{THz}$ and peaks around $0.15 \mathrm{THz}$. Although this measurement was obtained in less that a minute, we note that, in our setup, a measurement of a 25 ps long $\mathrm{THz}$ electric-field pulse is obtained in $20 \mathrm{~ms}$ with a dynamic range of 50 .

Figure 3 shows the measured peak $\mathrm{THz}$ electric field as a

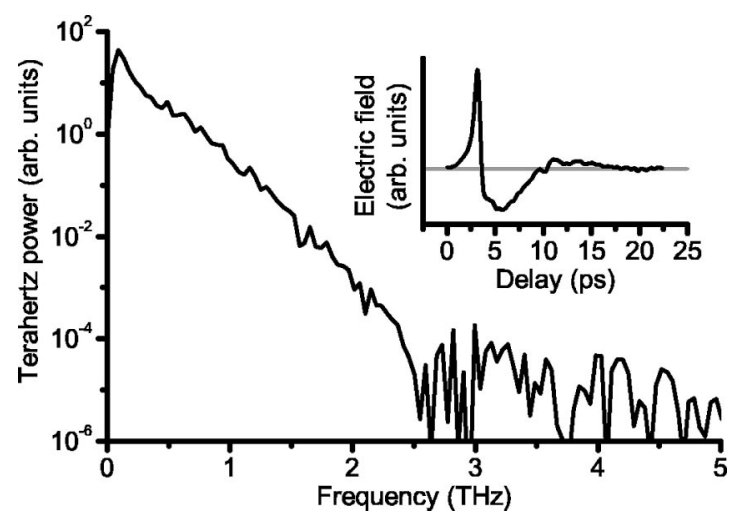

FIG. 2. THz power spectrum, calculated from the THz electric field as a function of delay shown in the inset. The measurement was performed with the tip pressed against the crystal, directly above the probe beam. This

measurement was obtained in less than a minute.
Downloaded 17 Aug 2010 to 131.180 .130 .114 . Redistribution subject to AIP license or copyright; see http://apl.aip.org/about/rights_and_permissions
FIG. 3. Measured peak THz electric field as a function of the tip-crystal distance. The inset shows a separate measurement of the region close to the crystal.

function of the tip-crystal separation. The measurement covers a total distance of about $30 \mu \mathrm{m}$. The inset of Fig. 3 shows an additional measurement, which zooms in on the region close to the crystal. We have chosen the $0 \mu \mathrm{m}$ position arbitrarily as the position where the signal reaches its maximum. Figure 3 shows that the $\mathrm{THz}$ electric field decreases to $30 \%$ of its maximum value, when the tip-crystal distance increases to $6.5 \mu \mathrm{m}$, which clearly indicates near-field behavior. When the tip is close to the crystal $(\sim 1 \mu \mathrm{m})$, it is abruptly attracted to the crystal, presumably by electrostatic forces, which gives rise to a sudden increase in the signal at $0 \mu \mathrm{m}$. The sharp, but smooth, decrease of the THz electric field when the distance to the crystal is increased only a few $\mu \mathrm{m}$, is related to results obtained by Knoll et al..$^{15}$ at the much shorter wavelength of $10 \mu \mathrm{m}$. They found that the scattering cross section of a tip close to a dielectric half space is strongly increased due to an image charge induced in the dielectric medium. It is likely, that these results also apply in our case, and that an increase of the scattering cross section is related to the strong enhancement of the local $\mathrm{THz}$ electric field when the tip approaches the crystal.

In Figs. 4(a)-4(d), we plot the integrated power of the $\mathrm{THz}$ pulses, measured while raster scanning the tip across the crystal surface, for four different tip-crystal separations. The four measurements clearly show a rapid increase in the $\mathrm{THz}$ spot size for increasing tip-crystal distances. This is quantitatively expressed by the results in Fig. 4(e), where we plot the measured $\mathrm{THz}$ spot size, defined as the full width at half maximum (FWHM) of the intensity, as a function of the tip-crystal distance. The $0 \mu \mathrm{m}$ point is chosen arbitrarily as the position where the spot size reaches its minimum. Figure 4(e) clearly shows that the spot diameter increases more than a factor of 6 when the tip-crystal separation increases to 30 $\mu \mathrm{m}$. This again strongly suggests that, close to the crystal, we are measuring in the near field of the tip. Figure 4 (e) also shows that subwavelength resolution is easily obtained even for relatively large tip-crystal distances. The smallest observed spot diameter is $18 \mu \mathrm{m}$. A spectral analysis (not shown here) clearly proves that the spot diameters are independent of frequency, with the smallest spot corresponding to a maximum resolution of $\lambda / 110$ at $0.15 \mathrm{THz}$.

Earlier measurements with a probe beam focal spot size of $50 \mu \mathrm{m}$ gave the smallest $\mathrm{THz}$ spot size of $50 \mu \mathrm{m}$. Our presently measured smallest spot size is $18 \mu \mathrm{m}$, again similar license or copyright; see http://apl.aip.org/about/rights_and_permissions 

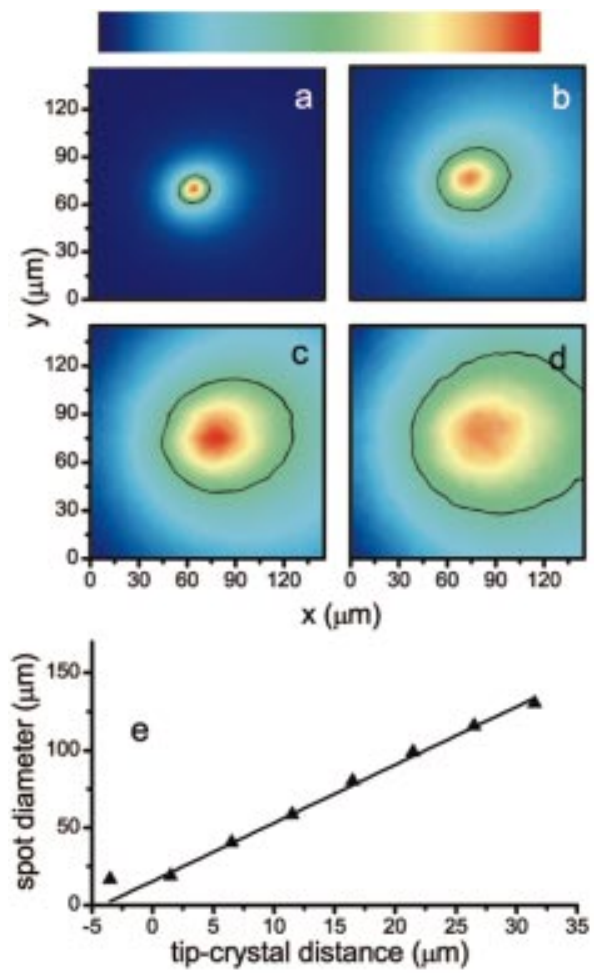

FIG. 4. (Color) THz intensity as a function of the $x y$ position of the tip along the crystal. The scans (a) and (b) are $50 \times 50$ pixel images with a 3 $\times 3 \mu \mathrm{m}^{2}$ pixel size, and (c) and (d) are $30 \times 30$ pixel images with a 5 $\times 5 \mu \mathrm{m}^{2}$ pixel size. In the pictures (a), (b), (c), and (d), the tip is positioned at $0,10,20$, and $30 \mu \mathrm{m}$ distance from the crystal, respectively. The color scale is shown at the top. The color levels run from zero (navy blue) to maximum intensity (red), and are separately normalized for each scan. The black line is the FWHM of the spot. Graph (e) shows the measured FWHM spot diameter as a function of tip-crystal distance. The solid line is a linear fit through all points but the first.

to the probe beam focal diameter of $20 \mu \mathrm{m}$, suggesting that the observed $\mathrm{THz}$ spot sizes are determined by the focal diameter of the probe. This indicates that a resolution better than $18 \mu \mathrm{m}$ is achievable in our setup if we focus our probe tighter. Ultimately, we envisage an experiment in which a thin sample is placed on the crystal underneath the tip. When the crystal is raster scanned underneath the tip, a twodimensional $\mathrm{THz}$ image of the sample is obtained. A high resolution, however, is only possible, if the physical size of the tip is smaller than the desired resolution. Ultimately, we expect that the resolution of our setup will reach about $1 \mu \mathrm{m}$, limited by the wavelength of our probe beam.

The advantages of our method to use THz TDS to produce near-field images are that it is coherent, intrinsically broadband, and uses only $40 \mu \mathrm{W}$ of input power. Coherent detection offers the advantage of measuring both amplitude and phase, while broadband detection allows us to obtain information on a wide range of frequencies in one measurement. The chance that a sample is heated significantly or even damaged is negligible in our setup, since we use low input powers. We note that the time-dependent nature of $\mathrm{THz}$ pulses makes it possible to use our setup for visible pump$\mathrm{THz}$ probe measurements on microscopic systems.

In conclusion, we have presented a method to obtain a subwavelength resolution in $\mathrm{THz}$ imaging by electrooptically measuring the $\mathrm{THz}$ electric field close to a sharp copper tip in a (100) oriented GaP crystal. We demonstrate a smallest $\mathrm{THz}$ spot size of $18 \mu \mathrm{m}$, determined by the probe beam diameter, and argue that our method should be able to reach a resolution of $1 \mu \mathrm{m}$, limited by the focal diameter of the probe beam.

Note added in proof. After submission of this manuscript, we have further improved our setup. Our presently measured smallest $\mathrm{THz}$ spotsize is now $8 \mu \mathrm{m}$.

The authors would like to thank W. T. Wenckebach for useful discussions. This work was performed as part of the EU TERAVISION Program (IST-1999-10154), and as part of the research program of the "Stichting voor Fundamenteel Onderzoek der Materie (FOM)," which is financially supported by the "Nederlandse Organisatie voor Wetenschappelijk Onderzoek (NWO)."

${ }^{1}$ D. Grischkowsky, S. Keiding, M. van Exter, and C. Fattinger, J. Opt. Soc. Am. B 7, 2006 (1990).

${ }^{2}$ R. A. Cheville and D. Grischkowsky, Appl. Phys. Lett. 67, 1960 (1995).

${ }^{3}$ Z. Jiang, M. Li, and X.-C. Zhang, Appl. Phys. Lett. 76, 3221 (2000).

${ }^{4}$ M. Walther, B. Fischer, M. Schall, H. Helm, and P. Uhd Jepsen, Chem. Phys. Lett. 332, 389 (2000).

${ }^{5}$ D. M. Mittleman, R. H. Jacobsen, and M. C. Nuss, IEEE J. Sel. Top. Quantum Electron. 2, 679 (1996).

${ }^{6}$ Z. Jiang, X. G. Xu, and X.-C. Zhang, Appl. Opt. 39, 2982 (2000).

${ }^{7}$ S. Mickan, D. Abbott, J. Munch, X.-C. Zhang, and T. van Doorn, Microelectron. J. 31, 503 (2000).

${ }^{8}$ M. Brucherseifer, M. Nagel, P. Haring Bolivar, H. Kurz, A. Bosserhoft, and R. Büttner, Appl. Phys. Lett. 77, 4049 (2000).

${ }^{9}$ O. Mitrofanov, I. Brener, R. Harel, J. D. Wynn, L. N. Pfeiffer, K. W. West, and J. Federici, Appl. Phys. Lett. 77, 3496 (2000).

${ }^{10}$ K. Wynne, J. J. Carey, J. Zawadzka, and D. A. Jaroszynski, Opt. Commun. 176, 429 (2000).

${ }^{11}$ Q. Chen and X.-C. Zhang, IEEE J. Sel. Top. Quantum Electron. 7, 608 (2001).

${ }^{12}$ G. Zhao, R. N. Schouten, N. van der Valk, W. T. Wenckebach, and P. C. M. Planken, Rev. Sci. Instrum. 73, 1715 (2002).

${ }^{13}$ P. C. M. Planken, H.-K. Nienhuys, H. J. Bakker, and W. T. Wenckebach, J. Opt. Soc. Am. B 18, 313 (2001).

${ }^{14}$ W. A. Kütt, W. Albrecht, and H. Kurz, IEEE J. Quantum Electron. 28, 2434 (1992).

${ }^{15}$ B. Knoll and F. Keilmann, Opt. Commun. 182, 321 (2000). 